\title{
Assessing abdominal aorta narrowing using computational fluid dynamics
}

\author{
Mohammad Al-Rawi ${ }^{1}$ Ahmed M. Al-Jumaily ${ }^{2}$
}

Received: 14 April 2014 / Accepted: 11 August 2015 / Published online: 29 August 2015

(C) International Federation for Medical and Biological Engineering 2015

\begin{abstract}
This paper investigates the effect of developing arterial blockage at the abdominal aorta on the blood pressure waves at an externally accessible location suitable for invasive measurements such as the brachial and the femoral arteries. Arterial blockages are created surgically within the abdominal aorta of healthy Wistar rats to create narrowing resemblance conditions. Blood pressure is measured using a catheter inserted into the right femoral artery. Measurements are taken at the baseline healthy condition as well as at four different severities (20, 50, 80 and $100 \%)$ of arterial blockage. In vivo and in vitro measurements of the lumen diameter and wall thickness are taken using magnetic resonance imaging and microscopic techniques, respectively. These data are used to validate a 3D computational fluid dynamics model which is developed to generalize the outcomes of this work and to determine the arterial stress and strain under the blockage conditions. This work indicates that an arterial blockage in excess of $20 \%$ of the lumen diameter significantly influences the pressure wave and reduces the systolic blood pressure at the right femoral artery. High wall shear stresses and low circumferential strains are also generated at the blockage site.
\end{abstract}

Keywords Arterial blockage $\cdot$ Pulse wave . Atherosclerosis · CFD

Ahmed M. Al-Jumaily

ahmed.al-jumaily@aut.ac.nz

http://www.ibtec.org.nz

1 Manukau Institute of Technology, Auckland, New Zealand

2 Institute of Biomedical Technologies, Auckland University of Technology, Private Bag 92006, City Campus, Auckland WD301B, New Zealand

\section{Introduction}

Pulsatile blood flow travels through the cardiovascular system to deliver essential substances of nutrients, oxygen and hormones to the body's organs. Any abnormal deformation or narrowing of the artery lumen causes patient discomfort, low substance circulation through the body and the subsequent deprivation of blood flow to organs is called ischaemia. Medical studies reported that this disease incidence is associated with diabetes, obesity, stress conditions, family history and smoking $[1,4,8,16,17,21]$. These factors can increase the likelihood of infections occurring at the artery wall and blockages developing due to the creation of clots by the endothelium layer to treat the infection [17]. However, whilst a blockage takes considerable time to develop, it may suddenly manifest (usually as an ischaemic attack) to a high risk of mortality. Therefore, identifying arterial blockage development at an early stage is extremely important, and an early diagnostic method could contribute significantly to the prevention of devastating health events associated with these diseases.

For diagnostic purposes, the general consensus among the medical and scientific community is the need for better understanding of the relationships between the onset and progression of arterial blockage and the wall shear stress (WSS) resulting from blood flow and the circumferential strain (CS) resulting from arterial wall stretching. Since blood flow and pressure waveforms are dependent on a number of characteristics (such as hemodynamic stresses) in addition to physiological conditions, clinical investigations demand comprehensive screening, which make them expensive and time-consuming. Computational fluid dynamic (CFD) simulation may be a preferable approach, as investigation can be performed with greater convenience, high speed and low cost, allowing prediction of the blockage in its early development. 
CFD methods have also been used to investigate the opposite scenario of arterial deformation. Soudah et al. [22] examined the relationship between hemodynamic factors and the rupturing of an abdominal aortic aneurysm (AAA). Their investigation was carried out based on converting 2D CT images for unhealthy patients to a 3D geometry, with a rigid wall assumption, using CFD methods. Their work showed that the development of AAA rupture is strongly correlated with the arterial geometrical and hemodynamic loads.

Since it is very difficult if not impossible to test CFD models using invasive clinical data collected from humans with the disease, animal models are adopted to justify the computational findings. The cardiovascular system of the Wistar strain rat has a lot of similarity to that of humans; thus, it is ideal to use this model to investigate cardiovascular diseases [1, 11, 13, 20, 22, 25-27] and produce predictable and controllable symptoms.

Animal experimental models have been used by biomedical engineers and scientists for a long time to enhance the understanding of diseases in humans, leading to the discovery of treatments to preserve human lives [5, 6, 18-20, 23]. Previous studies $[5,7,18,19]$ showed that animal models have resulted in meaningful investigations for early stages of disease development, although the selection of the animal was important to the disease under investigation.

The ideal animal for the investigation of cardiovascular disease in humans should bear reasonable physiological similarity to humans and produce symptoms which are predictable and controllable. The animal should also satisfy economical, technical and ethical welfare considerations. Wistar strain rats were deemed acceptable for the animal model in this investigation. They are considered suitable for cardiovascular and infectious diseases research [5, $7,18,19]$ and are the strain most frequently used for the investigation of cardiovascular diseases.

Previous studies have employed Wistar strain rats for similar investigations. Rentschler and Baxter [18] investigated the development of abdominal aortic aneurysm in rats in terms of the stresses affecting arterial wall compliance and stiffness which resulted in the development of a laboratory system for assessing the pressure-diameter correlation with nonlinear material properties of the artery wall. Larsen et al. [14] used hylan gel injected into the femoral artery, creating a clot, to simulate cardiovascular disease in rats. The experimental data in this study were gathered using a similar technique, but the degree to which the artery was blocked was varied using cable ties, rather than gel, to effect the narrowing of the artery.

This is a twofold paper. First, it intends to clinically determine the effect of the severity of aorta blockage on the blood pressure measured invasively at an accessible site such as the femoral artery. Second, based on the first part, this work intends to develop and validate a CFD model to determine the effect of this blockage on the WSS, CS and the blood pressure waveform at this site.

\section{Methodology}

\subsection{Animal model}

The clinical experiment is performed on 10 Wistar rats weighing 250-350 g following appropriate ethical protocols approved by the Auckland University of Technology Ethical Approval Committee (EAC). The EAC reviewed the application of this experiment in reference to the use of animals for research purposes and was satisfied that the animal use described therein was in compliance with the Animal Welfare Act 1999 and all other Acts of New Zealand Parliament, regulations and by-laws relating to performing experiments on animals. The animal data were collected at the Vernon Jansen Unit (VJU) at the University of Auckland, with ethical approval R915 approved on 15August 2011 and valid for 4 years. The researchers were in charge of all procedures performed on the animals and ensured that responsibilities towards the physical well-being, health and behavioural needs of animals were met, and that pain and distress were alleviated.

To generate an unhealthy condition, the design of this experiment is based on surgically creating an arterial blockage at the abdominal aorta of healthy animals. These rats are anaesthetized during the whole procedure, so hopefully experience no pain or distress. The preparation of the anaesthesia is a mixture of $0.75 \mathrm{~mL}$ ketamine, $0.5 \mathrm{~mL}$ xylazine and $0.75 \mathrm{~mL}$ water. This mixture is injected by an authorized technician at the intraperitoneal region with $0.2 \mathrm{~mL} / 100 \mathrm{~g}$ dosage using a syringe with $<26 \mathrm{~g}$ needle. The method of injection used a gentle manual restraint technique. The frequency of dosage is once; however, there is a second dose to top up after 45-60 min if needed [12, 15]. Once all the procedures are completed, the animals are euthanized via cervical dislocation without recovery from anaesthetic. The dead animals are dissected so that the extent of plug formation in the artery can be visualized.

Using plastic cable, in Fig. 1a, $4^{\circ}$ of blockage severity is created by reducing the diameter at the abdominal aorta of each rat. The blockage severity is defined as: (1) slight blockage around $20 \%$, (2) medium blockage around $50 \%$, (3) severe blockage around $80 \%$ and (4) full blockage around $100 \%$; see Table 1. At this stage of the research, the main objective is to see whether these blockages can be detected by measuring the pressure at a site which could be noninvasively accessible for future measurements. Therefore, precise values of these blockages are not critical as the notion is to observe the changes rather than to quantify them. 


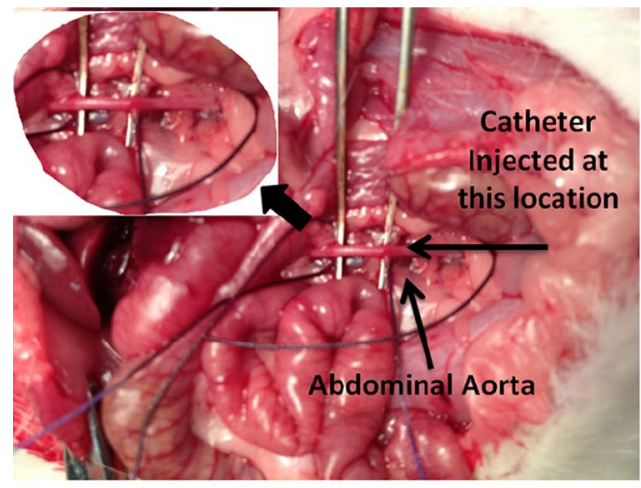

(a)

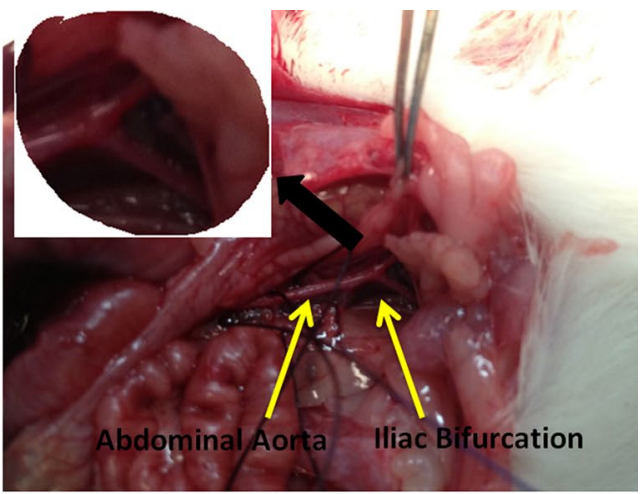

(b)

Fig. 1 a Plastic cable for creating arterial blockages at the abdominal aorta and $\mathbf{b}$ localizing the abdominal aorta, iliac bifurcation and right femoral artery

Table 1 Different controlled arterial blockages

\begin{tabular}{lcccc}
\hline Severity at abdominal aorta & $\sim 20 \%$ Severity 1 & $\sim 50 \%$ Severity 2 & $\sim 80 \%$ Severity 3 & $\sim 100 \%$ Severity 4 \\
\hline Diameter- $D(\mathrm{~mm})$ & $0.8 \pm 0.01$ & $0.5 \pm 0.01$ & $0.2 \pm 0.01$ & $0.0 \pm 0.01$ \\
Thickness- $t(\mathrm{~mm})$ & $0.1 \pm 0.009$ & $0.1 \pm 0.009$ & $0.1 \pm 0.009$ & $0.1 \pm 0.009$ \\
Length- $L(\mathrm{~mm})$ & $0.25 \pm 0.05$ & $0.25 \pm 0.05$ & $0.25 \pm 0.01$ & $0.25 \pm 0.05$ \\
\hline
\end{tabular}

The accessible site for the in vivo measurements could be the brachial or the femoral arteries; however, due to the animal biological constraints, the latter is used in this work. A small-lumen catheter (BD VialonTM ${ }^{\odot}$ Insyte 8360515, F1754-4 B9-09, Made in USA) is inserted at the right femoral artery to measure the blood pressure. This measurement is taken at the baseline (healthy condition) and then for each of the four severities. Figure $1 \mathrm{~b}$ shows the localization of both the abdominal aorta and the right femoral artery. This measurement is then used in the CFD model as an inlet boundary condition.

\subsection{Measurements}

Four types of measurements were made:

1. Blood pressure was measured at both healthy and unhealthy conditions in the same specimen using a custom-built diaphragm transducer (sample rate 5-10 kHz) attached to the catheter. This catheter has a bandwidth large enough to respond to pressure changes at high and low frequencies without loss of information, and its recorded signal is unaffected by changes in the signal frequency attenuated above $5 \mathrm{~Hz}$ (typical rat heart rate). The catheter and transducer were prefilled with heparinized saline and inserted into the blood vessel and then tied in place. Pressure fluctuations in the artery were then transmitted along the catheter tubing to the transducer's diaphragm, which moves in response. The diaphragm movements were converted into a varying electrical signal that was amplified through an ADInstruments Bridge Amplifier and recorded by a Power Lab system. Figure 2 shows typical pressure measurements for a rat in a healthy condition $(P-H)$ and in four different unhealthy conditions $(P-U)$. These measurements were used later as inlet and outlet boundary conditions for validating the CFD model.

2. For better accuracy and to avoid any variation in the artery dimensions which may occur after the animal was deceased, the artery geometry was measured using magnetic resonance imaging (MRI). Figure 3 shows the MRI measurements performed on a random rat

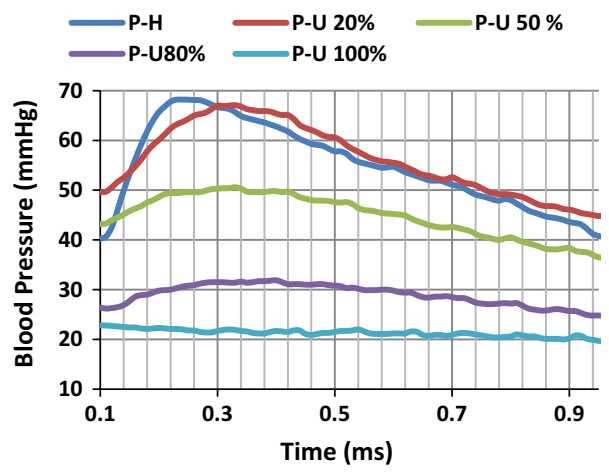

Fig. 2 Blood pressure measured over $1 \mathrm{~s}$ for a healthy specimen and four different arterial blockages ( $H$ healthy and $U$ unhealthy) 


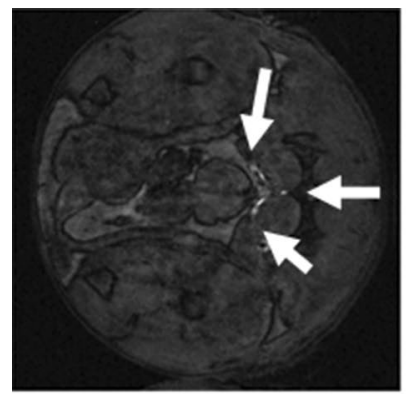

(a)

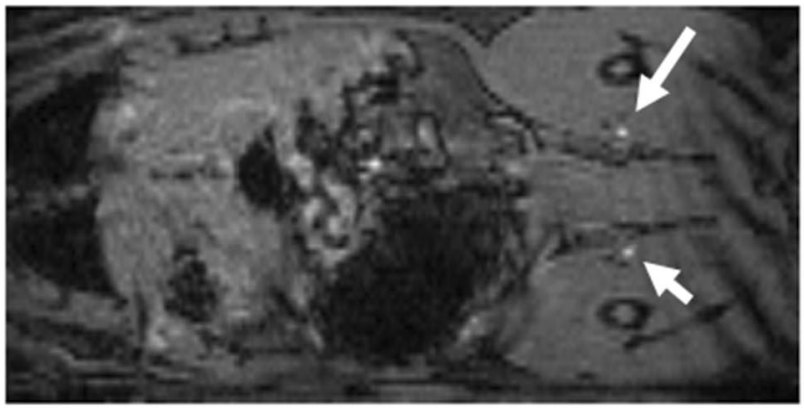

(c)

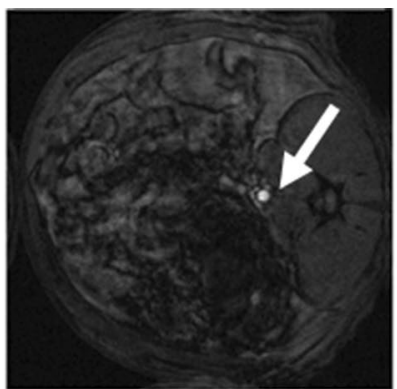

(b)

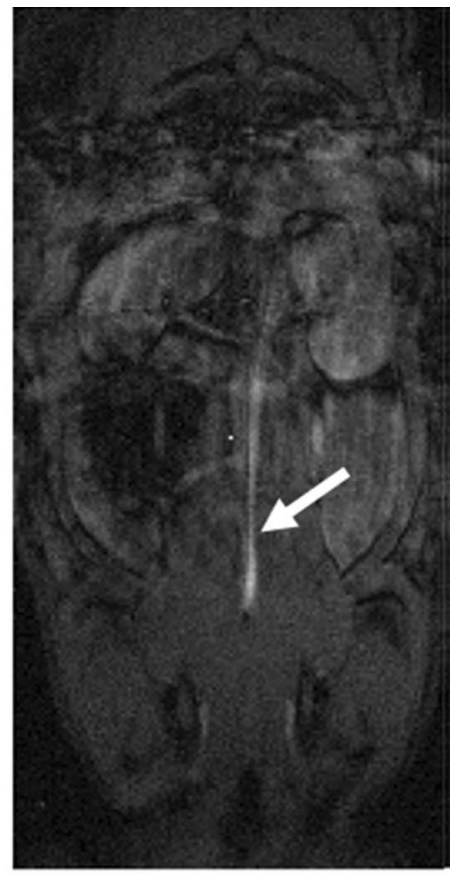

(d)

Fig. 3 MRI images for rat using MRI-4.7 T illustrating a cross-sectional image showing the iliac bifurcation, b abdominal aorta, $\mathbf{c}$ longitudinal section illustrating the right and left femoral arteries and $\mathbf{d}$ the abdominal aorta

using MRI-4.7 Tesla. Figure 3a shows the right and left femoral arteries, Fig. $3 \mathrm{~b}$ the iliac bifurcation crosssectional images, Fig. $3 \mathrm{c}$ the longitudinal section of the right and left femoral arteries and Fig. 3d the longitudinal section of the abdominal aorta.

3. Many studies [2, 3, 9] have used inaccurate assumptions for the mechanical properties in CFD modelling. To accurately reflect the nonlinear characteristics of the material properties in the current CFD modelling, a high-speed camera (Photron FastCam 1024PCI, Model $100 \mathrm{~K} \&$ Model KC AUX lens) was used to measure the actual arterial wall deformation for different severities, whilst the animal was alive and surgically open. Simultaneously, with these measurements, the pressure values at the aorta were determined using a catheter which was also inserted through the femoral artery. Figure 4 shows the speed camera application which has a function to measure arterial wall deformation at the pixel level; the scale is 1 pixel to $0.02 \mathrm{~mm}$.

4. After the animal was deceased, the aorta was dissected and then a microscopic technique was accurately used to determine the dimensional parameters of the abdominal aorta geometry, such as the inner and outer diameters, length and thickness. The rat was further investigated by measuring the length and diameters of the iliac bifurcation, and left and right femoral arteries. These measurements were then used to create the ide- alized geometry in the CFD models. Simulation of the geometry and its results were then validated and compared against the in vivo blood pressure values.

Table 2 shows the abdominal aorta measurements undertaken using the microscopic and MRI studies for the same randomly selected rat, which will be used to construct the 3D-CFD model.

\subsection{Mechanical properties}

Observations from the in vivo experiments indicate that there is a degree of dependence of the blood pressure values at the femoral artery on the shape of the abdominal aorta. Thus, the aorta lumen changes can be correlated with the blood pressure to develop a relationship which can be used to assess the degree of arterial blockage development at an early stage.

The deformable radius of the artery wall is determined from the speed camera images using the zoomin technique. This is then used to calculate the strain (strain $=\Delta r / r$ ). Using the aorta blood systolic pressure $P$, the stress is calculated from hoop stress $=P r / t$ and longitudinal stress $=\operatorname{Pr} / 2 t$, where $r$ represents the original radius of the artery which is measured from MRI experiments and checked with the microscopic measurements, $\Delta r$ represents the artery wall deformation and $\mathrm{t}$ wall thickness. Figure 5 


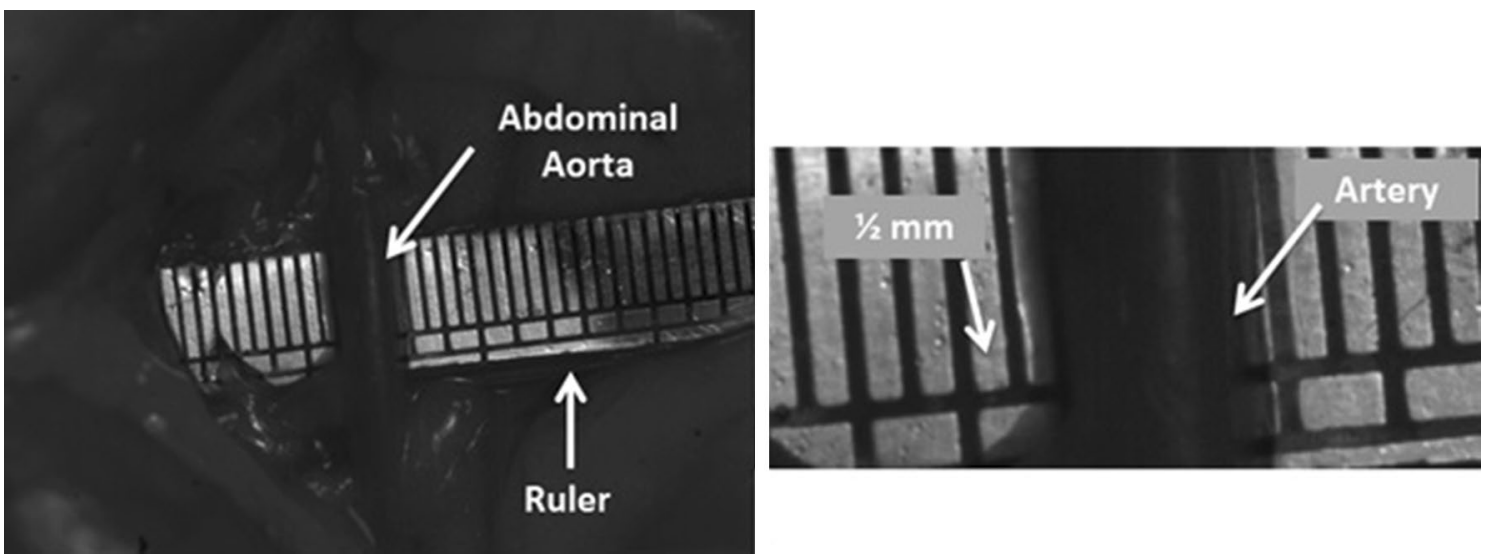

Fig. 4 Speed camera application for measuring deformation of the arterial wall in vivo experiment

Table 2 Measured in vitro data and in vivo

\begin{tabular}{lccc}
\hline Method & \multicolumn{2}{l}{ Microscopic/MRI } & \\
\cline { 2 - 4 } Location & Diameter $D(\mathrm{~mm})$ & Thickness $t(\mathrm{~mm})$ & Length $L(\mathrm{~cm})$ \\
\hline Abdominal aorta & $1.0 / 1.0 \pm 0.02$ & $0.1 / 0.09 \pm 0.001$ & $0.5 / 0.49 \pm 0.001$ \\
Iliac bifurcation & $1.0 / 1.0 \pm 0.01$ & $0.1 / 0.09 \pm 0.01$ & $0.5 / 0.48 \pm 0.02$ \\
Right femoral & $0.6 / 0.55 \pm 0.02$ & $0.09 / 0.09 \pm 0.001$ & $0.5 / 0.49 \pm 0.01$ \\
Left femoral & $0.6 / 0.58 \pm 0.02$ & $0.09 / 0.09 \pm 0.001$ & $0.5 / 0.49 \pm 0.01$ \\
\hline
\end{tabular}

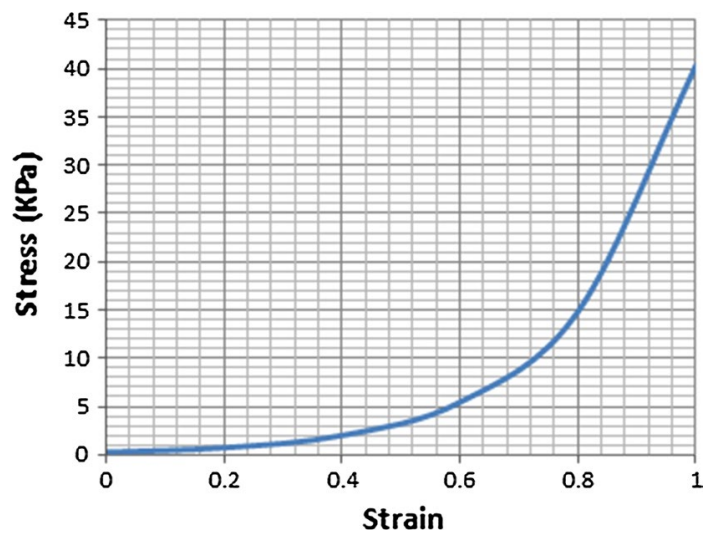

Fig. 5 Stress versus strain for the artery wall at the abdominal aorta

shows the stress-strain curve from which the modulus of elasticity $E$ is determined. The values of $E$ are determined at the abdominal aorta for the healthy condition and exhibited good agreement with those available in the literature [18]. Using Fig. 5, the nonlinear values are implemented as a nonlinear $E$ in the CFD model by entering the data of this graph into the material properties in ANSYS. The programme was designed to determine the corresponding value of $E$ for each value of the strain.

\section{Computational modelling}

The computational modelling of the arterial system is based on solving the theoretical dynamic models of blood flow and arterial geometries. Those theories have been developed based on idealized biomedical (cardiovascular) conditions such as introducing arterial narrowing for specific specimen geometry using ANSYS ${ }^{\circledR} 14.0$.

\subsection{Fluid-structure interaction}

Coupling between the blood and artery is due to the relatively low stiffness of the artery walls compared to compressibility of the fluid $[2,3,10,24]$. Hence, the pressure exerted by the flowing blood on the artery wall can result in considerable deformations of the artery. To address this coupling, a fluid-structure interaction (FSI) interface is developed using ANSYS $^{\circledR} 14.0$ to combine a constitutive model describing the stress distribution in the vessel's wall with the computational analysis of the blood flow in two-way FSI using the arbitrary Lagrangian-Eulerian (ALE) scheme in ANSYS ${ }^{\circledR}$ 14.0. The FSI solver is an automated tool which uses the ANSYS ${ }^{\circledR}$ Fluid Solver for the fluid domain and the ANSYS ${ }^{\circledR}$ TSM solver for the solid domain. The two solvers solve the equations for the fluid 


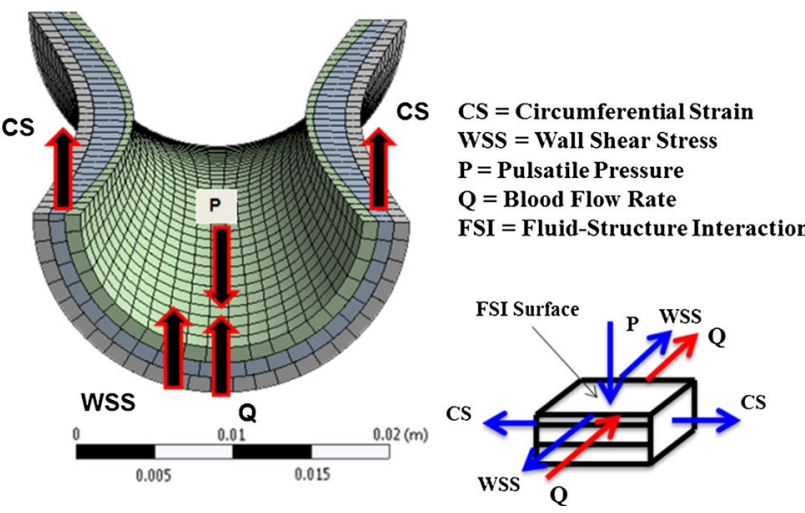

Fig. 6 Longitudinal section of the artery, demonstrating the WSS and CS stresses resultant from the blood $(P)$ and blood flow $(Q)$

and solid domains independently. The FSI solver transfers fluid forces (pressure) and velocities across the fluid-solid interface. The algorithm continues to loop through the solid and fluid analyses until convergence $\left(1 \times 10^{-4}\right)$ is reached for a given time step. Convergence in the stagger loop is based on the quantities being transferred at the fluid-solid interface.

This method allows for solving the fluid-solid phase coupled problems by accounting for both the instantaneous fluid mechanical forces on the artery wall and the effects of wall displacement on the fluid motion as shown in Fig. 6. This figure shows a schematic diagram of WSS which results from the blood pressure $(P)$ and CS which results from the arterial wall compliance and blood flow $(Q)$.

\subsection{Mesh generation}

Mesh generations of both the artery wall and the blood flow models are performed using the patch independent method. This method suited the size of the artery and the use of FSI. Other mesh options have been tried but did not achieve good skewness. After several attempts to select the mesh size, the best convergence was achieved by setting the number of elements and nodes in the abdominal aorta geometry for those models to: elements of range $(145,359$ $147,368)$ and nodes of range $(240,386-240,456)$. The blood geometries are meshed with elements 20,258 and nodes of 20,258 as shown in Fig. 7a-c. The skewness feature, available within ANSYS ${ }^{\circledR} 14.0$, is used to check and assess the mesh quality. The skewness measures the relative distortion of an element compared to its ideal shape on a scale from 0.0 (excellent) to 1.0 (unacceptable). Most meshes showed skewness value close to 0.8 ; however, the ones selected in the present models have skewness of 0.73 mesh quality which falls in the good range recommended by the software developers, see Fig. 7d.

\subsection{Material properties and boundary conditions}

The rheological properties for the blood are assumed to be in ranges that permit blood to be treated as an incompressible, homogeneous and Newtonian fluid. The dynamic viscosity of the blood is $0.004 \mathrm{Ns} / \mathrm{m}^{2}$, and density is $1100 \mathrm{~kg} / \mathrm{m}^{3}$.

The healthy artery wall is modelled assuming a hyperelastic Neo-Hookean model with Young's modulus obtained from Fig. 5 and Poisson's ratio of $v=0.45$.

The inlet and outlet boundary conditions were assumed to be blood pressure waveforms which were measured invasively as described in measurement section. This process is repeated for each rat individually to establish its boundary conditions.

In the computational model, a maximum number of 20 iterations were performed based on the level of residual reduction or absolute residual levels as a convergence criterion. The RMS residuals were reduced to below the target of $1 \times 10^{-4}$, and the pressure monitor points approached steady values.

The artery wall is treated as a flexible rubber pipe clamped at both ends. First, the steady-state FSI analysis is solved which is used as an initial boundary condition; then, waveforms of blood flow and pressure were at 0.2-s duration (the rat's heart rate is variable and may be taken at 5 beats/s), which represents several cycles per second. For the artery wall simulation, the settings of the steady solution are: auto-time stepping $=$ off; define by $=$ substeps; number of sub-steps $=1$; time integration $=$ off; and large deflection $=$ on. For the blood flow simulation, using ANSYS Multi-Field, adding the artery wall: coupling time duration $=1 \mathrm{~s}$; coupling time steps $=1 \mathrm{~s}$; and analysis type option $=$ steady state. The results of the steady state are used as initial conditions for the transient case, to achieve perfect convergence (with Residual Target 1.E-4 and Residual Type RMS): the maximum iterations $=5$ (number of CFX iterations per stagger loop); physical timescale $=0.1 \mathrm{~s}$ (for the steady-state solver only); the maximum coefficient loops $=10$; and the residual type $=$ maximum. For the external coupling: maximum iterations $=20$, and the under relaxation factor $=1$. The transient FSI response of the artery wall and blood flow is simulated following this set-up: coupling time duration $=20 \mathrm{~s}$; coupling time steps $=0.01 \mathrm{~s}$; and analysis type option $=$ transient. This means that where in the steady-state analysis $1 \mathrm{~s}$ of simulation "time" was completed, then setting the time duration of $20 \mathrm{~s}$ will provide another $19 \mathrm{~s}$ of simulation time for the transient analysis, as well as increasing the maximum iterations to 5 and the coefficient loops to 3 .

At each step, the blood flow is treated as incompressible and viscous. The flow regime is governed using the continuity and momentum equations (Navier-Stokes equations) which were solved in ANSYS-CFX using the finite volume 
Fig. 7 3D model of unhealthy abdominal aorta a the artery wall, $\mathbf{b}$ the blood body (top view), $\mathbf{c}$ the side view of the blood body and $\mathbf{d}$ skewness mesh element quality ranges (ANSYS ${ }^{\circledR} 14.0$ )

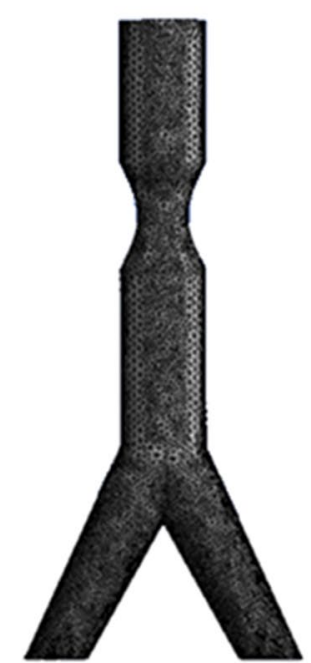

(a)

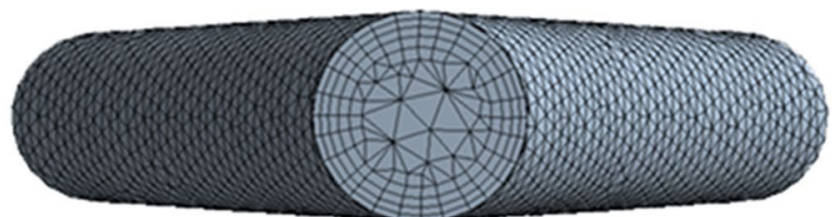

(b)

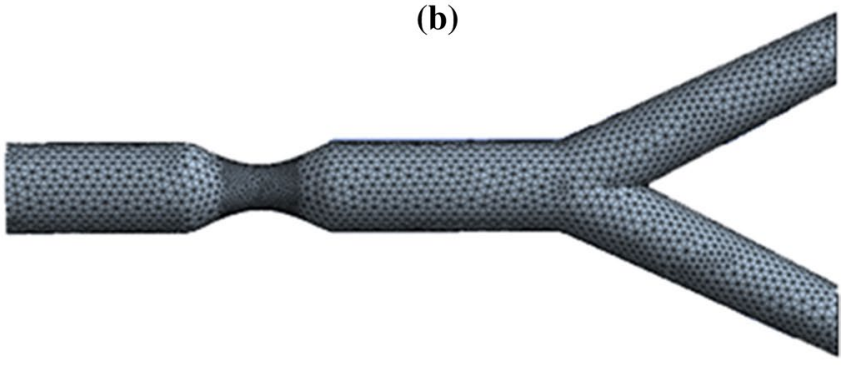

(c)
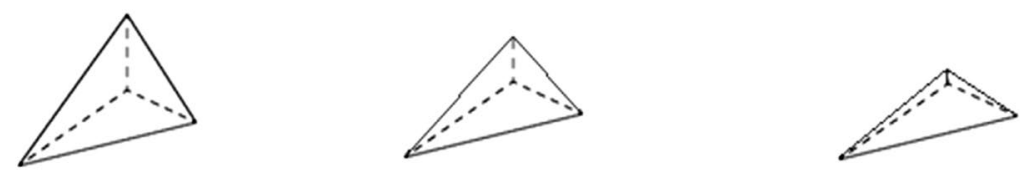

\begin{tabular}{|lccccc|}
\hline $0-0.25$ & $0.25-0.50$ & $0.50-0.80$ & $0.80-0.95$ & $0.95-0.98$ & $0.98-1.00$ \\
\hline Excellent & very good & good & acceptable & bad & Unacceptable \\
\hline
\end{tabular}

(d)

method. The fluid pressure determined at the interface of the blood and artery wall is then applied as a load on the wall to solve for the deformation of the artery in ANSYS transient structural analysis using the finite element method. The displacement obtained in the structural analysis is transferred back to ANSYS-CFX to adjust the fluid domain and update the blood flow analysis. This procedure continues until overall equilibrium is reached between the CFD solution and structural solution.

\section{Results}

The material properties for the artery wall were used, and the recorded experimental sets of data were implemented in the computational model as inlet and outlet boundary conditions to achieve realistic CFD analysis. A computational 3D-CFD model capable of representing pressure propagation based on animal arteries is developed, and this model is used to investigate the significance of different arterial blockage severities on the blood pressure measured at the abdominal aorta as well as at the femoral artery. To validate the model, the blood pressure values at the abdominal aorta from CFD/FE simulation are compared with those from the animal model, Fig. 8. These two values show good agreements with an average error of $0.94 \%$ based on iteration and time discretization errors; the two-way FSI ANSYS ${ }^{\circledR} 14$ scheme is very powerful in investigating the interaction between blood flow and artery wall deformation, which minimizes the average error. This high degree of agreement is attributed to the realistic material properties and boundary conditions obtained from the invasive data.

Figure 9 shows a comparison between the CFD/FE results and the in vivo data measured at the right femoral artery for one rat in healthy condition and in the four stages of blockage. For viable presentations of the experimental data, $1 \mathrm{~s}$ is used

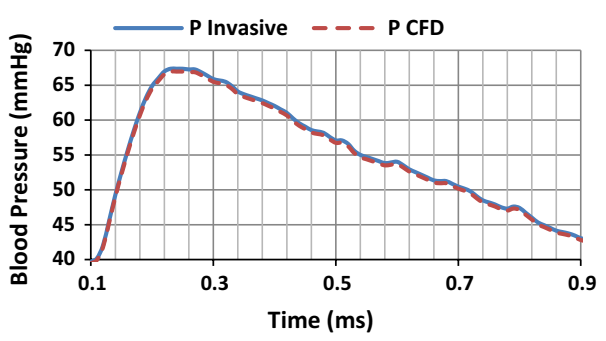

Fig. 8 Blood pressure measured invasively at the abdominal aorta compared to the CFD results 


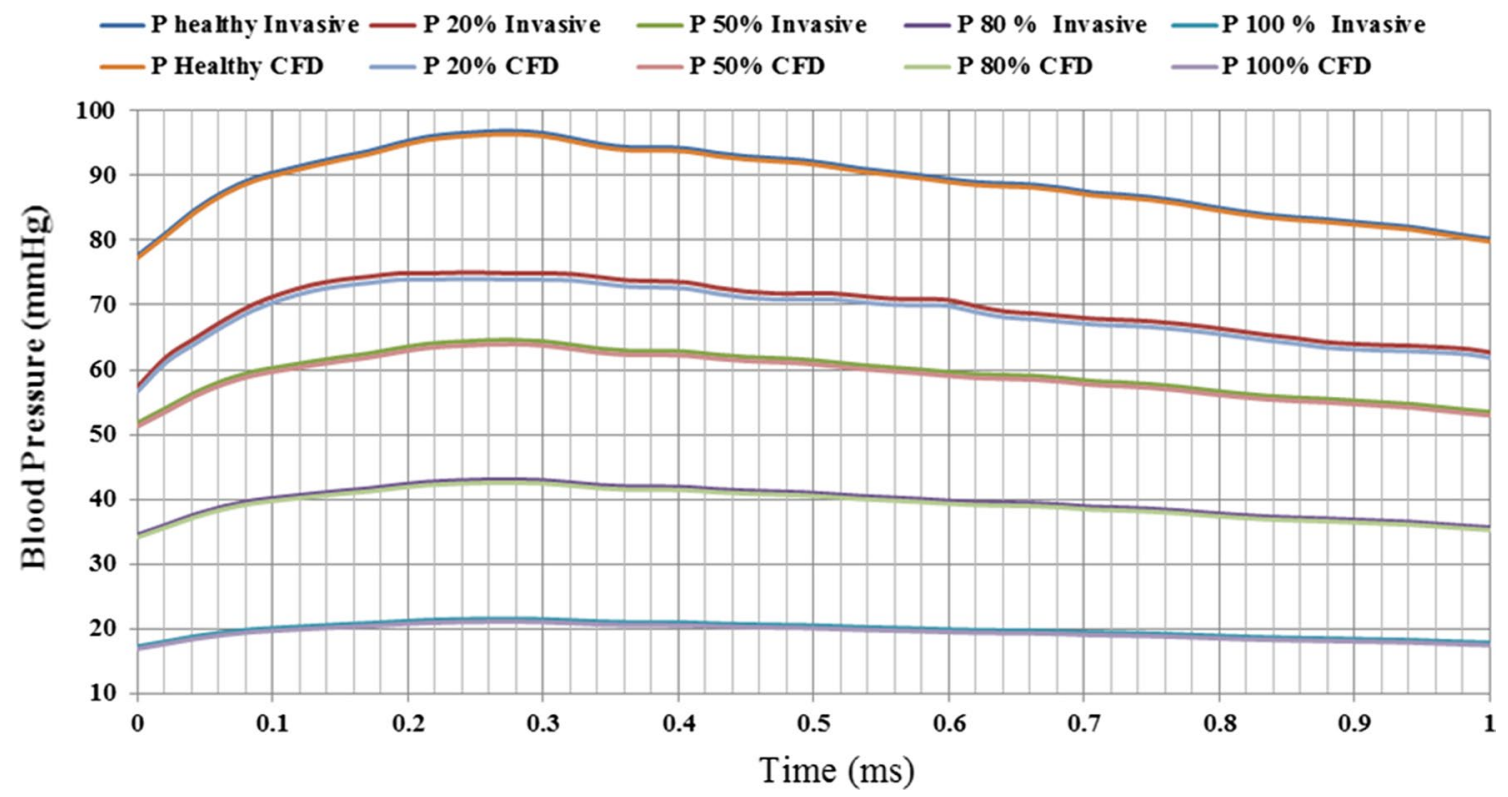

Fig. 9 Blood pressure at the right femoral artery for both the simulated (CFD) simulation results and the in vivo experiments

to compare the experimental and computational results. It is evident that these pressure values are affected by the degree of arterial blockage created at the abdominal aorta. These results show that decreasing the diameter of the abdominal lumen decreases the systolic and diastolic blood pressure measured at the right femoral artery. Although the later measurement was made at a local location, we expect this to reflect on the whole-blood circulation system. This may be attributed to the increase in kinetic energy and reduction in the blood pressure. In addition, these results show that the arterial compliance is related to the propagated pressure waves; as the artery wall stiffness increases, the pressure drops.

Figure 9 shows a minor error between the experimental and computational data which is encouraging for further investigation in using the CFD simulation to study the WSS and CS for the healthy condition as well as for the four arterial blockage conditions.

Using CFD modelling with the two-way FSI scheme, the WSS and CS are determined and presented in Fig. 10 at two locations (the abdominal aorta and the right femoral artery, which correspond to the locations of the experimental measurements). The figure shows that the arterial blockages amplify CS and reduce the WSS at the disease location along with decreasing the amplitude of pressure waves at the right femoral artery. The created blockage causes more reflections of the forward and backward blood pressure wave, and this requires the heart muscles to work harder to pump the blood through the circulatory system. This results in exhausting the arteries serving the heart and leads to a heart attack due to the overpowering of the blood flow inside it. The model accounts for this by correlating

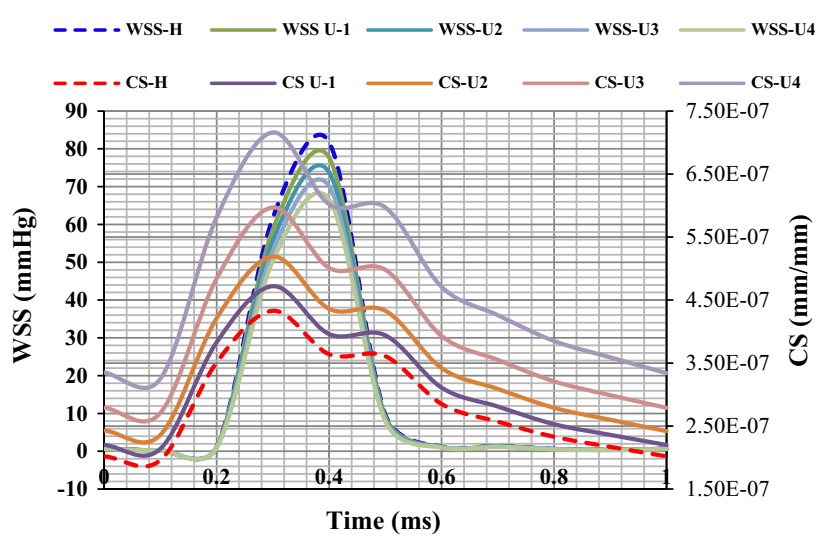

Fig. 10 WSS and CS for healthy and the development of arterial blockage at a the abdominal aorta and $(H=$ healthy and $U=$ unhealthy)

CS with the pulsatile blood flow and the pressure diameter response of the physical artery wall to the displacement vector of the computational grid points of the artery wall.

\section{Discussion}

To the best of our knowledge, the surgical procedure used to induce arterial blockage in an animal model is presented for the first time in this work. In addition, whilst previous publications assume the material properties of the artery wall to be linear, which is not anatomically representative, our model rectifies this by adopting nonlinearity. 
These realistic material properties and boundary conditions are introduced for both wall thickness and blood pressure waves and give rise to greater accuracy of the CFD model predictions of the complex physiological parameters associated with blood pressure wave propagation.

The animal experimental protocols were designed to create arterial blockages at the abdominal aorta. The blood pressure at the abdominal aorta and right femoral artery for both healthy and blocked conditions was measured and compared. The results show that increasing the blockage by $20 \%$ reduces the maximum amplitude of the pulse wave at the right femoral artery by $15-25 \%$ as compared with that measured at the abdominal aorta.

From medical perspectives, the risk of the disease becomes apparent to diagnose at the $50 \%$ blockage. Both Figs. 11 and 12 illustrate the CFD results for one rat, showing how the arterial blockage could be correlated with the hemodynamic properties. Figure 11 shows the contour profile for WSS at $50 \%$ lumen diameter severity at three different times of $0.3,0.5$ and $0.8 \mathrm{~ms}$ from the onset of the waveform. Figure 12 shows the CS for an arterial blockage of $50 \%$ at the abdominal aorta in terms of waveforms and contours selected at the same time of the WSS. The narrowing of the abdominal aorta is showing highest magnitude of WSS contour at the inner of the artery wall compared to the outer and significantly affecting its values along the iliac bifurcation and femoral arteries as seen at $0.3 \mathrm{~ms}$, as illustrated in Fig. 12. However, the magnitude of CS contour at the blockage site reduces at the outer artery wall compared to that of the inner wall for the 0.3-ms time domain. The results of WSS and CS magnitude at both 0.5 and $0.8 \mathrm{~ms}$ show no significant effect on further sites from disease location which indicates that the propagated backward waves are less effective at downstream locations. This may be attributed to the fact that the circulatory system at the right femoral artery is blocked by the catheter. Furthermore, the outcome of these two figures shows that high artery WSS is obtained with low CS at the blockage site compared to further locations, where the forward waves of the WSS are intensive, and could be measured to screen the development of disease.

The in vivo experiments presented in this study indicate that the arterial compliance and severity are inversely proportional to the blood pressure measurement at the right femoral artery. In other words, this study has indicated that WSS and CS significantly affect the artery wall, which is the main
Fig. 11 WSS for the FSI for atherosclerosis of $50 \%$ severity at the abdominal aorta at different times $(0.3,0.5$ and $0.8 \mathrm{~ms})$
Fig. 12 CS of the artery wall of $50 \%$ severity at the abdominal aorta at different times $(0.3,0.5$ and $0.8 \mathrm{~ms}$ )
WSS 0.3

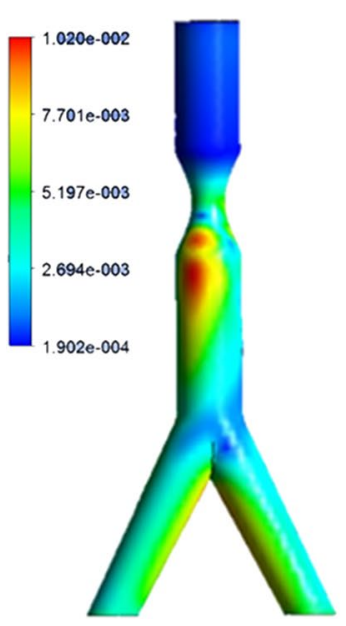

WSS 0.5

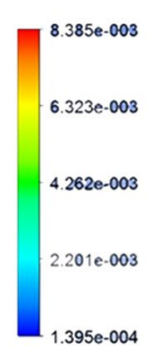

更
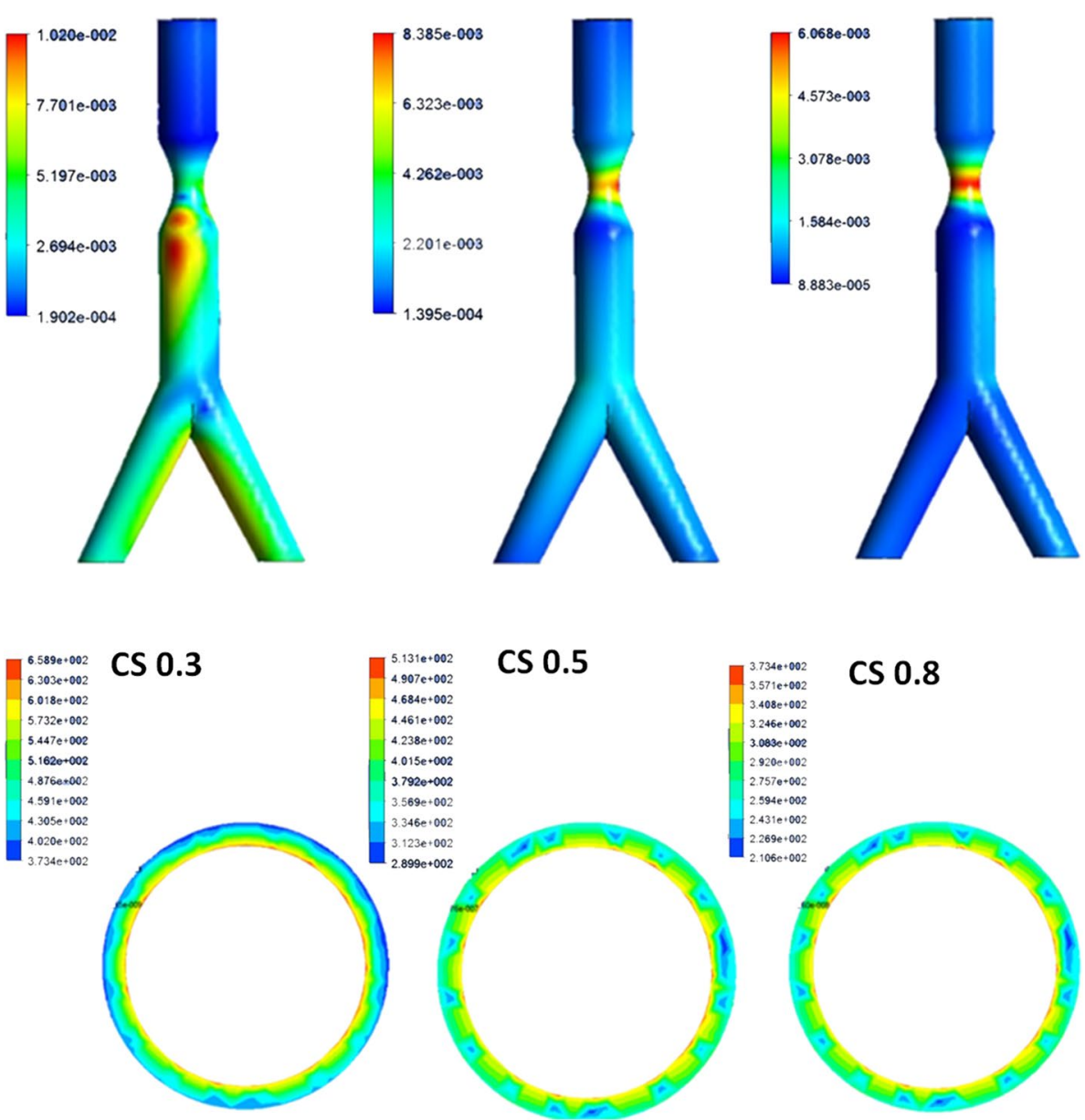

WSS 0.8

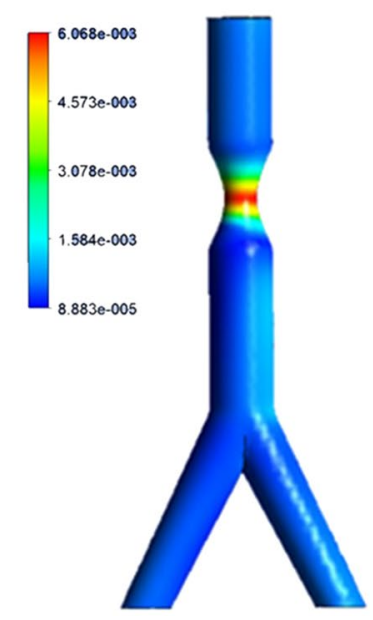




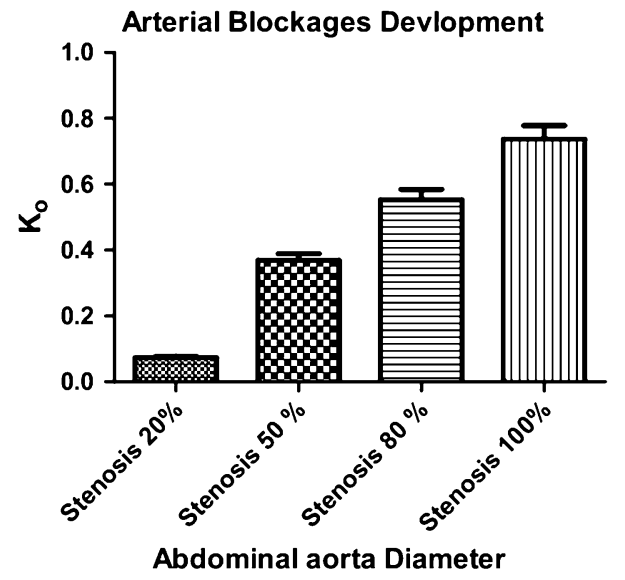

Fig. $13 K_{o}$ at the arterial blockages with different severities of 20, 50,80 and $100 \%$ for all ten rats (weighing $300-350 \mathrm{~g}$ )

cause of disease of the artery layers. However, presently, there is no clear medical evidence quantifying the range of changes in WSS for different cardiovascular diseases. Moreover, there is insufficient information on the critical values of this stress for diagnosing atherosclerosis and aneurysm medically.

The results are correlated by finding the coefficient $K_{o}=\frac{V}{P}$, where $V$ is the blood velocity and $P$ is blood pressure in terms of waveforms at the arterial blockage location, and could be used as an indication of arterial blockage development along the abdominal aorta. Figure 13 shows the increase in $K_{o}$ with increasing arterial narrowing in each specimen, for four severities of arterial blockages 20, 50, 80 and $100 \%$ created at the abdominal aorta. This graph is correlated with the arterial compliance which is calculated computationally from the CFD/FE analysis in Figs. 11 and 12.

\section{Conclusion}

This is a feasibility study to investigate surgically and computationally the WSS based on developing arterial blockages for the same specimen. This study compares between the healthy condition and the four different severities (20,50, 80 and $100 \%)$ of arterial blockages. Animal experimental data demonstrate that measuring the blood pressure at a distance from arterial blockage gives an acceptable indication of the blockage and its severity. The CFD model helps in generalizing this hypothesis and opens new directions for further investigation. Future work could also include the tissue pre-stress analysis, to correlate between the WSS and CS and the development of the disease.

Acknowledgments This study was supported and funded by IBTec (Institute of Biomedical Technologies), Auckland University of Technology. The animal data were collected at Vernon Jansen Unit (VJU) at the University of Auckland with ethical approval R915 and the assistance of Dr. Jun Lu and IBTec's PhD Candidate Miguel Jo-Avila.
There are no known conflicts of interest associated with this publication, and there has been no significant financial support for this work that could have influenced its outcome.

\section{References}

1. Abassi Z, Goltsman I, Karram T, Winaver J, Hoffman A (2011) Aortocaval fistula in rat: a unique model of volume-overload congestive heart failure and cardiac hypertropy. J Biomed Biotechnol 2011:1-13. doi:10.1155/2011/729497

2. Bathe M (1999) Kamm RD (1999) A fluid-structure interaction finite element analysis of pulsatile blood flow through a compliant stenotic artery. J Biomech Eng 121:361-369

3. Cebral JR, Castro MA, Putman CM (2005) Numerical simulation of flow alterations after carotid artery stenting from multimodality image data. In: Third MIT conference on computational fluid and solid mechanics, pp 607-611

4. Chan WC, Wright C, Riddell T, Wells S, Kerr AJ, Gala G, Jackson R (2008) Ethnic and socioeconomic disparities in the prevalence of cardiovascular disease in New Zealand. N Z Med J 121(1285):11-20

5. Charvatova Z, Ostadalova I, Zicha J, Kunes J, Maxova H, Ostadal B (2012) Cardiac tolerance to ischemia in neonatal spontaneously hypertensive rats. Physiol Res 61(Suppl. 1):S145-S153

6. Daghero F, Bueno N, Peirone A, Ochoa J, Torres GF, Ganame J (2008) Coarctation of the abdominal aorta an uncommon cause of arterial hypertension and stroke. Circ Cardiovasc Imaging 1:e4-e6

7. Doggrell SA, Brown L (1998) Rat models of hypertension, cardiac hypertrophy and failure. Cardiovasc Res 39(1998):89-105

8. Ebrahim S, Montaner D, Lawlor DA (2004) Clustering of risk factors and social class in childhood and adulthood in British women's heart and health study: cross sectional analysis. BMJ 328(7444):861. doi:10.1136/bmj.38034.702836.55

9. Gao F, Ohta O, Matsuzawa T (2008) Fluid structure interaction in layered aortic arch aneurysm model assessing the combined influence of arch aneurysm wall stiffness. Australas Phys Eng Sci Med 31:32-41

10. Gerbeau J-F, Vidrascu M, Frey P (2005) Fluid-structure interaction in blood flows on geometries based on medical imaging. Comput Struct 83(2005):155-165

11. Gianluca DS, Peter M, Mathieu DB, Patrick S, Pascal V, Bendict V (2010) Patient-specific computational fluid dynamics: structured mesh generation from coronary angiography. Med Biol Eng Compu 48(4):371-380

12. Hedenqvist $\mathbf{P}$ (2008) Anaesthesia and analgesia for surgery in rabbits and rats: a comparison of the effects of different compounds. Ph.D. thesis, Karolinska Institutet, Stockolm, Sweden

13. Langeveld B, Roks AJ, Tio RA, VanBoven AJ, Van der Want JJ, Henning RH, Van Beusekom HM, Van der Giessen WJ, Van Gilst WH (2004) Rat abdominal aorta stenting: a new and reliable small animal model for in-stent restenosis. J Vasc Res 41:377-386

14. Larsen NE, Leshchiner EA, Parent EG, Hendrikson-Aho J, Balazs EA (1991) Hylan gel composition for percutaneous embolization cardiovascular. J Biomed Mater Res 25(1991):699-710

15. Marshall S, Milligan A, Yates R (1994) Experimental techniques and anaesthesia in the rat and mouse. Anzccart Fact Sheet $7(1): 1-4$

16. Mendis S (2005) Cardiovascular risk assessment and management in developing countries. Vasc Health Risk Manag 1(1):15-18 
17. Nichols WW, O'Rourke MF (2011) McDonald's blood flow in arteries. Hodder Arnold, London

18. Rentschler ME, Baxter BT (2008) Screening aortic drug treatments through arterial compliance measurements. Curr Vasc Pharmacol 6:250-257. doi:10.2174/157016108785909751

19. Restini C, Reis R, Costa-Neto C, Garcia-Cairasco N, Cortesde-Oliveira J, Bendhack L (2012) Role of endothelium on the abnormal Angiotensin-mediated vascular functions in epileptic rats. J Biophys Chem 3(2):174-182

20. Russell JC (2003) Of mice and men, rats and atherosclerosis. Cardiovasc Res 59(2003):810-811

21. Sebastia C, Quiroga S, Boye R, Perez-Lafuente M, Castella E, Alvarez-Castells A (2003) Aortic stenosis: spectrum of diseases depicted at multi-section CT. Radio Graph 23:S79-S91

22. Soudah E, Ng EY, Loong TH, Bordone M, Pua U, Narayanan S (2013) CFD modelling of abdominal aortic aneurysm on hemodynamic loads using a realistic geometry with CT. Comput Math Methods Med 2013:9, Article ID 472564

23. Still WJS, O'Neal RM (1962) Electron microscopic study of experimental atherosclerosis in the rat. Am J Pathol 40(1):21-35

24. Tang D, Yang C, Kobayashi S, Ku DN (2001) Steady flow and wall compression in stenotic arteries: a three-dimensional thick-wall model with fluid-wall interactions. J Biomech Eng 123:548-557

25. Vukicevic AM, Stepanovic NM, Jovicic GR, Apostolovic SR, Filipovic ND (2014) Computer methods for follow-up study of hemodynamic and disease progression in the stented coronary artery by fusing IVUS and X-ray angiography. Med Biol Eng Comput 52(6):539-556. doi:10.1007/s11517-014-1155-9

26. Wronska-Nofer T, Szendzikowski S, Oberebska-Parke M (1980) Influence of chronic carbon disulphide intoxication on the development of experimental atherosclerosis in rats. $\mathrm{Br} \mathbf{J}$ Ind Med 37:387-393

27. Yannis P, Nicolas A, Ioannis S, Mohmmad IK, Georgios CB, Elena E, Andreas SA (2013) Effect of head posture on the healthy human carotid bifurcation hemodynamics. Med Biol Eng Compu 51(1-2):207-218

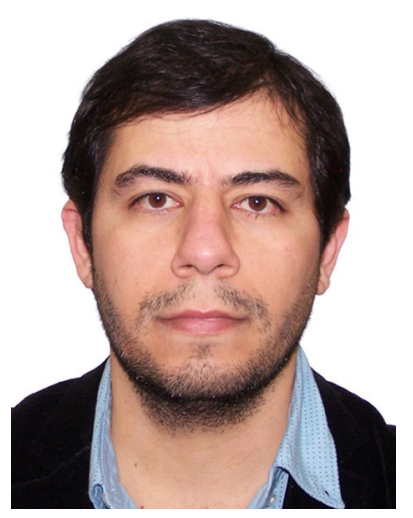

Dr. Mohammad Al-Rawi focuses his research on using computational fluid dynamics (CFD) to develop noninvasive tools for detecting cardiovascular diseases. An animal model was used for in vitro and in vivo experiments to validate the CFD results. Currently, Dr. Al-Rawi is a senior lecturer/programme leader, School of Engineering at the Manukau Institute of Technology, Auckland, New Zealand. His academic experience is primarily in the field of Mechanical and Biomedical

Engineering research and teaching Applied Computational Modelling, Fluid Mechanics and Strength of Materials.

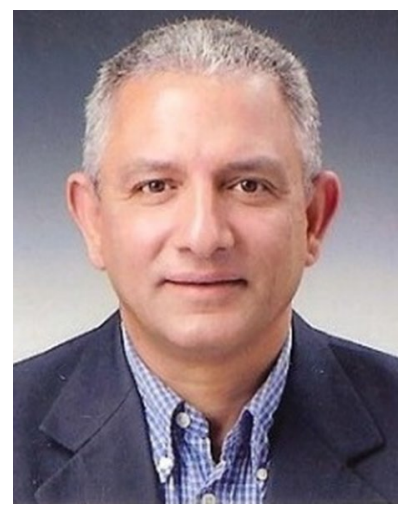

Ahmed M. Al-Jumaily completed $\mathrm{PhD}$ and $\mathrm{MSc}$ from the Ohio State University and BSc from the University of Baghdad. $\mathrm{He}$ is a FELLOW member of the ASME and a member of 11 other international professional societies. He is Editor of the ASME monograph series Biomedical and Nanomedical Technologies and the Editor in Chief of the Journal of Biomedical Engineering and Technology and has been on the editorial and refereeing boards for several international journals. $\mathrm{He}$ has published more than 270 papers in international journals and conference proceedings including two ASME books on Vibration and Acoustics in Biomedical Applications and a third one on CPAP devices and supervised more than 90 postgraduate students in biomedical applications, vibrations, biomechanics and electroactive polymers. During his academic career, he has forged strong alliances between academia and industries, in particular in the medical devices area. His current research focuses on biomedical applications with particular interest in the application of vibration and acoustics to airways constriction therapies and artery noninvasive diagnostics. 
Medical \& Biological Engineering \& Computing is a copyright of Springer, 2016. All Rights Reserved. 